\title{
Journal of Pharmacy
}

\section{Potential Nanospray Inhalation of Remdesivir and Hydroxychloroquine using Poly (lactic-co-glycolic) Acid as Fast Delivery for Covid-19 treatment.}

\author{
Muhammad Taher $^{1 *}$, Siti Syazwani Shaari ${ }^{1}$ and Deny Susanti ${ }^{2}$
}

\section{ABSTRACT}

Introduction: The oral medication of remdesivir and hydroxychloroquine face several limitations in covid-19 therapy. Despite having the first-pass metabolism, it also has a limitation in the patient who has hospitalised with a severe covid-19 infection. It is especially for a drug that is targeting the angiotensin-converting enzyme II (ACE2) receptor where the receptors are found abundantly in the lung, kidney, heart, and gastrointestinal tract. Therefore, an alternative delivery such as nanospray inhalation would provide a great benefit to those patients.

Methods: Scientific sources from Scopus, PubMed, Google Scholar, EBSCO, ScienceDirect, and Elsevier were accessed for publication of this review article regarding the nanospray inhalation for Covid-19.

Results: Since the main organ infected by SARS-CoV-2 is the trachea and lung, inhalation may be the best route to deliver the drug to the site of action. It is proposed that poly (lactic-co-glycolic) acid to be used in the formulation.

Conclusion: Poly (lactic-co-glycolic) acid (PLGA) is considered a suitable polymer since it is biocompatible and noncytotoxic, it is the most widely applied in drug delivery either as carrier or excipient for the optimal formulation and distribution of the drugs. Dry powder inhalation of remdesivir and hydroxychloroquine may be an alternative way to deliver the drug against Covid- 19 .

\section{ARTICLE HISTORY:}

Received: 30 August 2020

Accepted: 17 December 2020

Published: 8 January 2021

\section{KEYWORDS:}

COVID-19, nanospray, hydroxychloroquine, remdesivir, inhalation, antiviral.

\section{HOW TO CITE THIS ARTICLE:}

Taher, M., Shaari, S. S. \& Susanti, D. (2021). Potential Nanospray Inhalation of Remdesivir and Hydroxychloroquine using Poly (lactic-co-glycolic) Acid as Fast Delivery for Covid19 Treatment. Journal of Pharmacy, 1(1), 34-44.

\section{*Corresponding author:}

Email address: mtaher@iium.edu.my Tel:+609 5704842

Fax: +6095716775

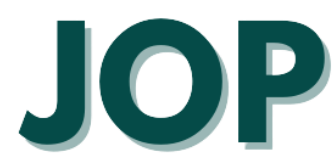

\begin{abstract}
Authors' Affiliation:
1 Department of Pharmaceutical Technology, Kulliyyah of Pharmacy, International Islamic University Malaysia, Jalan Sultan Ahmad Shah, 25200 Kuantan, Pahang, Malaysia.

${ }^{2}$ Department of Chemistry, Kulliyyah of Pharmacy, International Islamic University Malaysia, Jalan Sultan Ahmad Shah, 25200 Kuantan, Pahang, Malaysia.
\end{abstract}




\section{Introduction}

In December 2019, the world has been threatened with a recent coronavirus outbreak caused by Severe Acute Respiratory Syndrome Coronavirus 2 (SARS-CoV-2) which is believed to be originated from a seafood market in Hubei Province, Wuhan in central China (Al-Tawfiq et al., 2020; Morse et al., 2020; She et al., 2020). However, the seafood market is not the only source of the new coronavirus as 13 out of 41 cases in China were not linked to the market (She et al., 2020). This new coronavirus has caused the pandemic of pneumonia in humans (She et al., 2020; Wang et al., 2020) and causes severe respiratory failure requiring ICU admission (Bouadma et al., 2020). The disease, known as coronavirus disease 2019 (COVID19), had been declared by the World Health Organization (WHO) as a pandemic in just 45 days from the onset of the new coronavirus emergence, indicating significant public health issues around the world (Panati \& Narala, 2020). The virus not only affected global public health but also local economies (Liu et al., 2020). On January 12, 2020, the World Health Organization (WHO) had named the new coronavirus as the 2019-novel coronavirus (2019-nCoV). Disease caused by this new coronavirus was named by the WHO as Covid-19 on 11 February 2020, the same day of the Coronavirus Study Group (CSG) of the International Committee proposed to name the new coronavirus as Severe Acute Respiratory Syndrome Coronavirus 2 (SARS-CoV-2) (Guo et al., 2020).

As of December 31, 2019, 104 cases were diagnosed and the Covid-19 infection was extended to all the 34 districts of China including Macau, Hong Kong, and Taiwan in just 30 days. By February, the confirmed case increased rapidly to $2,175,567$ with 2239 deaths reported in China, and 1151 cases were confirmed with 8 losses reported in 26 countries ( Zhou et al., 2020a). Recently, the death rate of $2019-\mathrm{nCoV}$ worldwide is $6.93 \%$ with 265,084 deaths reported (Covid-19 Visualiser) compared to $9.6 \%$ of SARS-CoV and $34.4 \%$ of Middle East Respiratory Syndrome Coronavirus (MERS) (She et al., 2020). As of May 12, 2020, the number of deaths worldwide is 303,371 (Covidvisualizer.com). It is estimated that the basic reproductive figure for Covid-19 infection lies between 2.2 and 4.8 (Bouadma et al., 2020; G. Zhou et al., 2020). Thus it is believed that the increase in the widespread of SARS-CoV-2 infection is due to genetic variation and regular recombinant of the viral genomes as well as increased human and animal activities (She et al., 2020).

Generally, coronaviruses (members of the family Coronaviridae) are RNA viruses with features of enveloped, single-stranded and positive-sense, (Guo et al., 2020; She et al., 2020), which able to spread to birds and all mammals including humans, thus affecting the body systems such as respiratory, enteric, hepatic, and neurologic (She et al., 2020). It was reported that the genome sequence of SARS-CoV-2, SARS-CoV, MERS$\mathrm{CoV}$ is $96.2 \%, 75-80 \%$, and $40 \%$ similar to the betacoronaviruses detected in bats respectively (Guo et al., 2020; She et al., 2020; Wang et al., 2020). The SARS-CoV and MERS-CoV are both fatal coronaviruses that occurred in different areas in 2002 and 2012 respectively (She et al., 2020). Meanwhile, SARS-CoV-2 shares $82 \%$ similarity of the sequence identity with the SARS-CoV (Morse et al., 2020; Wang et al., 2020). Because of the comparable genomic sequence, the bat was allegedly to be the natural host of the virus originated in which the virus is spread by an unknown intermediate host from bats to humans (Guo et al., 2020).

In the early of the pandemic, remdesivir and hydroxychloroquine have been suggested as possible prevention or treatment of covid-19 based on evidence of in vitro inhibition of SAR-Cov-2. The current available formulation of remdesivir and hydroxychloroquine are not suitable for critically infected patients, therefore, we would like to provide a brief insight regarding an alternative preparation for critically ill patients.

\section{Methods}

Information on the nanospray inhalation for Covid-19 was obtained via a literature search conducted for publications using various electronic databases, such as PubMed, Google Scholar, International Islamic University Malaysia IIUM EBSCO, ScienceDirect, and Elsevier. The publications selected for this review is ranging from 2000 to 2020 to make sure the information gathered is validated and updated. The keyword 'SARS-CoV-2' and 'Covid-19' were used as primary searches, while terms such as nanospray, inhalation, and antiviral were applied as secondary searches. As there was a limited source on invivo and in-vitro studies related to nanospray inhalation for Covid-19 at the time of writing this article, the only scientific theory regarding nanospray inhalation from the selected literature search was included to highlight the therapeutic use of nanospray inhalation for Covid-19. Literature search on potential medications to be delivered as nanospray inhalation such as remdesivir and hydroxychloroquine were also included. Therefore, this review article is not exhaustive to medications used for Covid-19 that cannot be delivered through nanospray inhalation technology.

\section{Transmission and Clinical Presentation}

As mentioned before, Covid-19 is a disease that is spread from an unidentified animal source to a human and it is transmissible from one person to another (Bouadma et al., 2020). SARS-CoV-2 is transmitted primarily through respiratory droplets generated after coughing, sneezing, and speaking (Itani et al., 2020) as well as through direct contact with an infected person for a low infective dose 
(Guo et al., 2020). The symptomatic or asymptomatic infected person may transmit the SARS-CoV-2 to a healthy person who is in close contact, or when he or she touches an infected surface and subsequently touches his or her face especially at the area of eyes, nose, or mouth (Itani et al., 2020). Family members, such as relatives and friends having in direct contact with patients or incubation carriers, are at high risk of infection transmission (Guo et al., 2020). Although the SARS-CoV-2 droplets can move less than 2 meters and do not stay in the air, a study found that the SARS-CoV-2 can remain sustainable in the aerosols for up to 3 hours, and on copper, cardboard as well as on plastic or stainless steel for 4 hours, 24 hours and, 2 to 3 days respectively (Itani et al., 2020).

The incubation period is mainly between 3 to 7 days (within 14 days) (Guo et al., 2020; Itani et al., 2020; She et al., 2020) and it is contagious during the latency period (Guo et al., 2020). There are no specific symptoms but the most frequent ones are an increase in body temperature, cough, exhaustion, sputum production, breathlessness, sore throat, and headache (Guo et al., 2020; She et al., 2020). Some patients may experience gastrointestinal symptoms (diarrhea and vomiting) (Guo et al., 2020). If the disease is uncontrolled, it can develop severe complications including acute respiratory distress syndrome (ASDS), septic shock, multiple organs failure, or even death (Guo et al., 2020; Itani et al., 2020; She et al., 2020). The most susceptible people to severe diseases are older patients ( $>65$ years old) and people with comorbidities such as hypertension, chronic obstructive pulmonary disease, diabetes, and cardiovascular disease (Guo et al., 2020; Itani et al., 2020).

\section{Site of Action}

It was found that the SARS-CoV-2 multiplies better in epithelial cells of the primary human airway than in normal tissue-culture cells as compared to the SARS-CoV and MERS-CoV (She et al., 2020) and it utilizes angiotensin-converting enzyme- 2 (ACE-2) receptor (Figure 1), just like SARS-CoV, to infect human being (Guo et al., 2020; She et al., 2020; G. Zhou et al., 2020). ACE- 2 can be found in type II alveolar cells of the lung and other epithelial cells located at the heart, kidney, ileum, esophagus, and bladder (Zhou et al., 2020). The receptors are abundant in lung alveolar epithelial cells and enterocytes of small intestines (Guo et al., 2020). The coronaviruses use their spike protein to attach to a host cell receptor for entry (Morse et al., 2020). Structural analysis showed that the spike of SARS-CoV-2 glycoprotein has a higher binding affinity towards the ACE-2 receptor (Morse et al., 2020; G. Zhou et al., 2020). The S-glycoprotein of the SARS-CoV-2 has 2 subunits; S1 and S2. S1 is responsible for evaluating the number of virus-hosts and cell tropism with receptor-binding domain (RBD), while $\mathrm{S} 2$ is responsible for the fusion of virus into cell membrane through a pair of domains, heptad repeats 1 (HR1) and (HR2) (Guo et al., 2020). Besides that, the SARS-CoV-2 also requires two proteins which are coronavirus main proteinase (3CLpro) and the papain-like protease (PLpro) to mediate the formation of new virions through the proteolysis process and one protein which is replicate or RNA-dependent RNA polymerase (RdRp) to replicate once it enters the human epithelial cells (Morse et al., 2020).

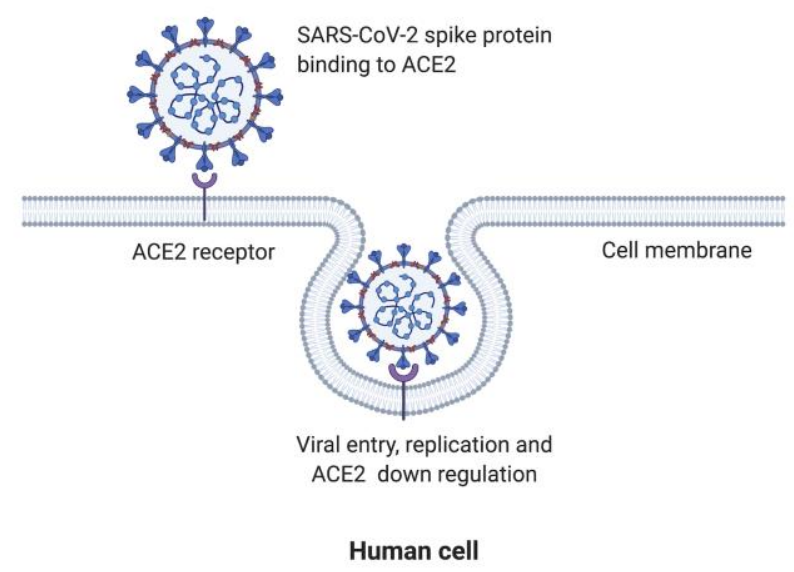

Figure 1. Typical binding of SARS-CoV-2 to ACE2 receptor. ACE2 receptor is an entry point by the virus to propogate inside the human cells.

\section{Treatment Approaches}

Currently, there is no definite treatment or vaccine available in the market to kill the SARS-CoV-2 (Bouadma et al., 2020; Panati \& Narala, 2020; She et al., 2020; Wang et al., 2020). In fact, in clinical practices, supportive therapy, oxygen therapy, or mechanical ventilation are used to prevent complications or reduce symptoms of the Covid-19 disease associated with respiratory disorders (She et al., 2020). It was reported that the SARS-CoV-2 invades the human body through its entry via ACE-2 receptors which are expressed on the epithelial cells of the lung, intestines, kidney, and blood vessels (Panati \& Narala, 2020). Targeting directly the ACE-2 receptors using ACE inhibitors or angiotensin-receptor antagonists had led to high morbidity in a patient with diabetes and hypertension as the drugs promote overexpression of the ACE-2 receptors, making them more favorable situation for the virus to invade the body system (Gaurav \& Ramarao, 2020; Panati \& Narala, 2020). Due to that, several drugs and treatment options were undergoing clinical trials and being tested for their efficacy against Covid 9 disease. Among them, remdesivir (an experimental drug) and hydroxychloroquine are the most frequent drugs that have been tested extensively (Gaurav 
\& Ramarao, 2020) due to their efficient action in impeding SARS-CoV-2 infection in-vitro (Morse et al., 2020).

Remdesivir, a novel antiviral drug, and chloroquine, an anti-malarial drug, were found to be effective in preventing the current novel coronavirus at an effective concentration (EC) of $1.1 \mu \mathrm{M}$ (Colson et al., 2020). Remdesivir is a prodrug and an analogue of a nucleoside. It was produced by Gilead Sciences located in the United State of America (USA) (Liu et al., 2020). In the earliest case report, the first Covid-19 patient from the USA had shown an improvement in his clinical condition after being treated with intravenous remdesivir (Guo et al., 2020; Holshue et al., 2020; Liu et al., 2020; She et al., 2020). To block the Covid-19 infection, remdesivir may inhibit the viral receptor-binding domain (RBD)-ACE-2 interaction by using peptides and their cocktails derive from RBD and ACE-2 (Morse et al., 2020). Meanwhile, chloroquine was commonly used to treat malarial infection and autoimmune disease, but nowadays, it has been reported that this drug has a wide-spectrum activity against viral infection. It can prevent virus infection by raising the endosomal $\mathrm{pH}$ necessary for virus and cell fusion, as well as obstructing the glycosylation of cellular ACE-2 receptors by SARS-CoV (Wang et al., 2020).

Since ACE-2 receptors are abundant in lung epithelial cells and its associated with respiratory problems among infected patients with SARS-CoV-2, this article review focuses to target directly the lungs through localised therapy by using nanospray inhalation. This formulation can produce an ideal particle size of a drug (about 1-5 $\mu \mathrm{m}$ ) which can efficiently reach the deep lung, allowing its penetration and deposition in the alveolar (Arpagaus et al., 2018; Karathanasis et al., 2005). This formulation is also able to avoid the first-pass metabolism and allow for more rapid onset of therapeutic action (Bartolucci, 2017). Thus, the lung which is infected by SARS-CoV-2 would be more reachable with pulmonary delivery compared to oral and intravenous injection. Besides that, poly(lactic-co-glycolic acid) (PLGA) will be used as a polymer since it is biocompatible, noncytotoxic, and widely used as carrier or excipient in drug delivery to achieve the best formulation and delivery of the drugs (Arpagaus, 2019a).

\section{Properties and Clinical Trials of Remdesivir and Hydroxychloroquine}

\section{Remdesivir}

Remdesivir (GS-5734), is an investigational nucleoside analog prodrug that has not yet approved or licensed anywhere including by the Food and Drug Administration (FDA) of the United States (US) (AlTawfiq et al., 2020; Guo et al., 2020; Ko et al., 2020). It is a small molecule with a molecular weight of $602.6 \mathrm{~g} / \mathrm{mol}$ and its chemical formula is $\mathrm{C}_{27} \mathrm{H}_{35} \mathrm{~N}_{6} \mathrm{O}_{8} \mathrm{P}$ (Ko et al., 2020). It was manufactured by Gilead Sciences in 2017 to treat the Ebola virus that was occurred in 2016 (Al-Tawfiq et al., 2020; Eastman et al., 2020; Gordon et al., 2020). Besides, it was reported that remdesivir can treat various RNA viruses including coronaviruses like SARS-CoV and MERS-CoV which were discovered in 2017. Currently, it has been investigated as one of the promising medications for SARS-CoV-2 infection (Eastman et al., 2020). This drug demonstrated wide antiviral activities such as inhibit human and zoonotic coronavirus (including SARS-CoV-2 and Ebola virus) in vitro and produce preventive and therapeutic effects in animal models of MERS-CoV and SARS-CoV infections ( Zhou et al., 2020).

Inside the cell, the prodrug remdesivir (GS-5734) is metabolised into adenosine nucleotide analogue (GS441524). Since the nucleotide analogue, GS-441524 is not highly cell-permeable, it requires di- and triphosphorylation to generate active nucleoside triphosphate (NTP) (Eastman et al., 2020; Ko et al., 2020; Sheahan et al., 2017). This is because the initial phosphorylation of nucleotide analogue into monophosphate form, is quite polar, so it could not diffuse back through the cell membrane and being trapped in the cells (Eastman et al., 2020). The NTP then will be misintegrated into the RNA viral via the viral RNA-dependent RNA polymerases $(\mathrm{RdRp})$ during genome replication thus suppressing viral RNA synthesis (Eastman et al., 2020; Gordon et al., 2020; Ko et al., 2020). The NTP resembles adenosine triphosphate (ATP) (Gordon et al., 2020). The NTP confuses the viral RdRp by working as an incorporation competitor with adenosine triphosphate (ATP) to enter into the nascent chain of viral RNA and results in immature cessation (Wang et al., 2020).

A case report associated with the earlier confirmed case of Covid-19 in the United States (US) has proven the efficacy of remdesivir to combat SARS-CoV-2. Intravenous remdesivir was given on the $7^{\text {th }}$ day to an infected Covid-19 patient following reports on the development of severe pneumonia. Surprisingly, the patient's medical status showed a positive outcome on the following day with no observed adverse effects. This finding has encouraged other researchers to conduct several clinical studies for the use of remdesivir in dealing with Covid-19 disease (Holshue et al., 2020). On the other hand, three out of twelve positive Covid-19 patients had been given with remdesivir treatment for 4-10 days, in which $200 \mathrm{mg}$ IV was administered on the first day, and $100 \mathrm{mg}$ for each subsequent day. It was noted that all the treated patients encountered "transient gastrointestinal symptoms, such as nausea, throwing up, gastroparesis, or rectal bleeding" after the initial dose. However, the therapy proceeded until there was an amelioration in the respiratory symptoms. Nevertheless, this finding is omitted from clinical effectiveness or health review due to the limited number of sample study involvement and lack of controlled randomisation (Eastman et al., 2020). 
In China, a phase 3 randomized, quadruple-blind, placebo-controlled clinical trial was enrolled at Capital Medical University, to determine the safety and efficacy of remdesivir in 308 hospitalised and infected adult patients with manifestations of mild to moderate symptoms of Covid-19 infection (NCT04252664). On the next day, another phase 3 clinical trial (NCT04257656) was enrolled at the same university to measure the efficacy and safety of remdesivir in 452 hospitalised patients with severe SARS-CoV-2 respiratory disease. All the participants involved in both trials were randomly administered with either placebo or remdesivir $200 \mathrm{mg}$ loading dose on the $1^{\text {st }}$ day followed by $100 \mathrm{mg}$ intravenous once-daily as maintenance doses for the following 9 days. After 28 days of both treatments, the patients' conditions such as fever, oxygen saturation, and respiratory rate became normal and there was also alleviation of cough for about 72 hours (AlTawfiq et al., 2020; Eastman et al., 2020).

To further evaluate the safety and efficacy of remdesivir, several phases 3 clinical studies were launched on infected Covid-19 patients around the world. Among the studies are NCT04292899, NCT04292730, NCT04280705, NCT04323761, ISRCTN83971151, NCT04315948， 2020-001052-18, NCT04321616, NCT04314817, NCT04302766 (Eastman et al., 2020) and NCT04252664 (Itani et al., 2020). However, since remdesivir is an experimental drug, it would be insufficient to be used for dealing with a huge number of patients promptly. Thus, another choice of potential drugs for large-scale use is chloroquine as it is largely available with proven safety record as well as relatively low cost (Liu et al., 2020).

\section{Chloroquine (CQ) and Hydroxychloroquine (HCQ)}

Chloroquine (4-aminoquinoline) is known as a drug used to treat malarial infection and autoimmune disease, and now, has been reported as a possible antiviral drug with broad-spectrum activity (O'Neill et al., 1998; Wang et al., 2020). It was synthesised by Hans Andersag, in 1934, under German chemical and pharmaceutical company Bayer AG (Thomé et al., 2013). It is a safe and cheap drug that has been used for more than 70 years (Gao et al., 2020; Wang et al., 2020). CQ with the chemical structure $\mathrm{C}_{18} \mathrm{H}_{26} \mathrm{CIN}_{3}$ is a small molecule and has a molecular weight of $319.872 \mathrm{~g} / \mathrm{mol}$ (O'Neill et al., 1998). In 1946, the first derivate of CQ, hydroxychloroquine (HCQ) sulfate was synthesised by inserting a hydroxyl group into chloroquine and exhibited lesser $(\sim 40 \%)$ toxic than chloroquine in animals (Liu et al., 2020; O'Neill et al., 1998). Both drugs, CQ, and HCQ are widely available as anti-inflammatory agents for treating rheumatoid arthritis and lupus erythematosus (Gao et al., 2020; Liu et al., 2020; Moore, 2020). These drugs are weak base drugs with similar chemical structures (Liu et al., 2020) and mechanisms of action as antiviral, anti-inflammatory, and immunomodulator. Thus, chloroquine and hydroxychloroquine may effective in treating patients with COVID-19 pneumonia (Gao et al., 2020; Wang et al., 2020).

Both CQ and HCQ interfere with the fusion of SARS$\mathrm{CoV}$ with the ACE-2 receptors on cells via de-acidification of lysosomes, thereby preventing cathepsins that require acidic conditions for optimal SARS-CoV spike protein cleavage (Gao et al., 2020; Liu et al., 2020; Singh et al., 2020). Originally, chloroquine (CQ) is lipophilic and in unprotonated form. It diffuses passively through cell membranes and into endosomes, lysosomes, and Golgi vesicles; where it becomes di-protonated (doubly positively charged), trapping the chloroquine in the organelle and elevating the surrounding $\mathrm{pH}$. The resultant increased in the $\mathrm{pH}$ value of the endosomes prevents viral replication including fusion and uncoating (Colson et al., 2020; Thomé et al., 2013; Warhurst et al., 2003). CQ and HCQ do not affect the level of ACE-2 expression on cell surfaces, but instead, they prevent the viral entry by inhibiting terminal glycosylation of ACE-2 receptor (target receptor for SARS-CoV and SARS-CoV-2 to enter the body), making it less efficient to interact with the SARS-CoV-2 spike protein (Thomé et al., 2013).

A group of researchers from China had previously conducted an in-vitro study to compare the antiviral effect of HCQ and CQ on VeroE6 cells infected with SARS$\mathrm{CoV}-2$ infection. The outcome showed that both drugs can efficiently inhibit COVID-19 infection (by reducing viral replication) with conventional dosing because of its high distribution in tissues as well as in the lung. They inhibited the entry, transport, and post-entry of SARS-CoV-2 into the cells. It was also reported that HCQ is less potent and less toxic than CQ in combating SARS-CoV-2 (Liu et al., 2020; Singh et al., 2020). This is because the hydroxyl group in the HCQ that makes it less permeable to the blood-retinal barrier thus lessen the risk of retinal toxicity (Singh et al., 2020). However, HCQ has a low selectivity index (SI) as compared to CQ thus, careful designing and safe management of the SARS-CoV-2 infection is required (Liu et al., 2020).

Besides, in vivo evaluation using infected SARS-CoV2 VeroE6 cells had been conducted to assess the effects of seven potential drugs including remdesivir and CQ on the cytotoxicity, virus production, and the rate of virus infection. At low-micromolar concentration, both remdesivir $\left(\mathrm{EC}_{50}: 0.77 \mu \mathrm{M}, \mathrm{EC}_{90}: 1.76 \mu \mathrm{M}\right)$ and $\mathrm{CQ}\left(\mathrm{EC}_{50}\right.$ : $\left.1.13 \mu \mathrm{M}, \mathrm{EC}_{90}: 6.9 \mu \mathrm{M}\right)$ effectively hindered the SARS$\mathrm{CoV}-2$ infection. It was known that remdesivir blocked at post-stage virus entry meanwhile chloroquine blocked both entry and following-entry of the SARS-CoV-2 infection in the VeroE6 cells. The antiviral activity of CQ is also enhanced with the synergy effect of its immunemodulating activity (Wang et al., 2020). Interestingly, in 
another study, it has been reported that the serum level of HCQ sulfate in humans is between 1.4-1.5 $\mu \mathrm{M}$ when given at doses of $6-6.5 \mathrm{mg} / \mathrm{kg} /$ day. This suggests that high distribution and concentration of HCQ sulfate in the liver, spleen, kidney, and lung could be achieved to inhibit SARS-CoV-2 infection (Liu et al., 2020).

To evaluate the efficacy and safety of CQ and HCQ, several ongoing clinical trials were currently conducted. The clinical trials can be tracked by the following numbers: NCT04303299, NCT04303507, NCT04304053, NCT04304053, NCT04286503, NCT04307693, NCT04261517, and NCT04308668 (Singh et al., 2020).

\section{Nanospray Inhalation}

The coronaviruses use their spike protein on its surface to recognise and bind to the angiotensin-converting enzyme (ACE)-2 receptor (abundant in the lung epithelial cells) of the host cell causing respiratory tract infection (Yang \& Wang, 2020). This gives the idea that local delivery of inhaled drugs which is usually used to treat lung diseases such as asthma, chronic obstructive pulmonary disease (COPD), and cystic fibrosis (Ungaro et al., 2012) may be necessary to treat SARS-CoV-2 infection. Lungs offer a unique route for localised therapy because of the large surface area of the alveolar region, the equivalence of inhalation to arterial injection, reasonably low proteolysis activity in the alveolar space, and lack of first-pass hepatic metabolism (Karathanasis et al., 2005; Ungaro et al., 2012). Inhalation of powder particles with a size below $5 \mu \mathrm{m}$ can be directly and efficiently delivered into the lower part of the respiratory tract (Ahmad et al., 2015; Beck-Broichsitter et al., 2012; Karathanasis et al., 2005; Ungaro et al., 2012). This local type of treatment provides fewer systemic effects than oral therapy (Ungaro et al., 2012).

Spray-dried powder is a suitable dosage form for the delivery of drugs through inhalation. It can be produced by a spray drying technique which has been an important technique for the pulmonary delivery of pharmaceutical proteins (Haggag \& Faheem, 2015). Spray drying is onestep, rapid, reproducible, and scalable solvent evaporation system that is suited to convert a solution, suspension, or emulsion into controlled size and shape of dried powder particles (Arpagaus, 2012, 2018, 2019b) (Figure 2). It is widely used in the pharmaceutical sectors for processing excipient, microencapsulation, or granulation (Arpagaus, 2018). There are two types of spray dryers available in the market nowadays; standard spray dryer and nanospray dryer. A standard spray dryer uses a pneumatic nozzle system while a nanospray dryer uses a piezoelectric system to produce liquid feed droplets by spraying the starting liquid material containing the drug into a hot drying medium (Patrizia et al., 2014). A standard spray-drying procedure comprises of four steps which are 1) atomisation of the liquid feed, 2) evaporating of the liquid feed through the drying gas, 3) formation of a particle, and lastly 4) separation and collection of the formed particle (Arpagaus, 2019b; Schafroth et al., 2012).

Interestingly, the innovative nano spray dryer has become a more promising technology to produce powder particles for inhalation as compared to standard spray dryers because of its ability in controlling and reducing powder particles (Patrizia et al., 2014). Nanospray dryer could produce liquid droplets using a piezoelectric system that functions at a certain ultrasonic frequency $(60 \mathrm{kHz})$ and thus forming a mist of droplets that has a remarkable ultra-fine particle size. The final dried particles eventually have an electrostatic charge which enables them to be deposited on the surface of the collecting electrode (Arpagaus, 2012; Schafroth et al., 2012). The uniqueness about this new spray dryer is that it utilises a gentle laminar airflow to minimise sample loss. Besides, it has a spray head device consists of a stainless-steel membrane. This membrane has several specific micron-sized holes (spray meshes with hole sizes of 4.0, 5.5, or $7.0 \mu \mathrm{m}$ ) to generate small particles (size ranging from 3 to $15 \mu \mathrm{m}$ ) in a very narrow size distribution. Last but not least, the novel spray dryer has an electrode collector that collects particles electrostatically charged to produce high yields and thus reduce particle wastages (Arpagaus, 2012; Patrizia et al., 2014; Schafroth et al., 2012).

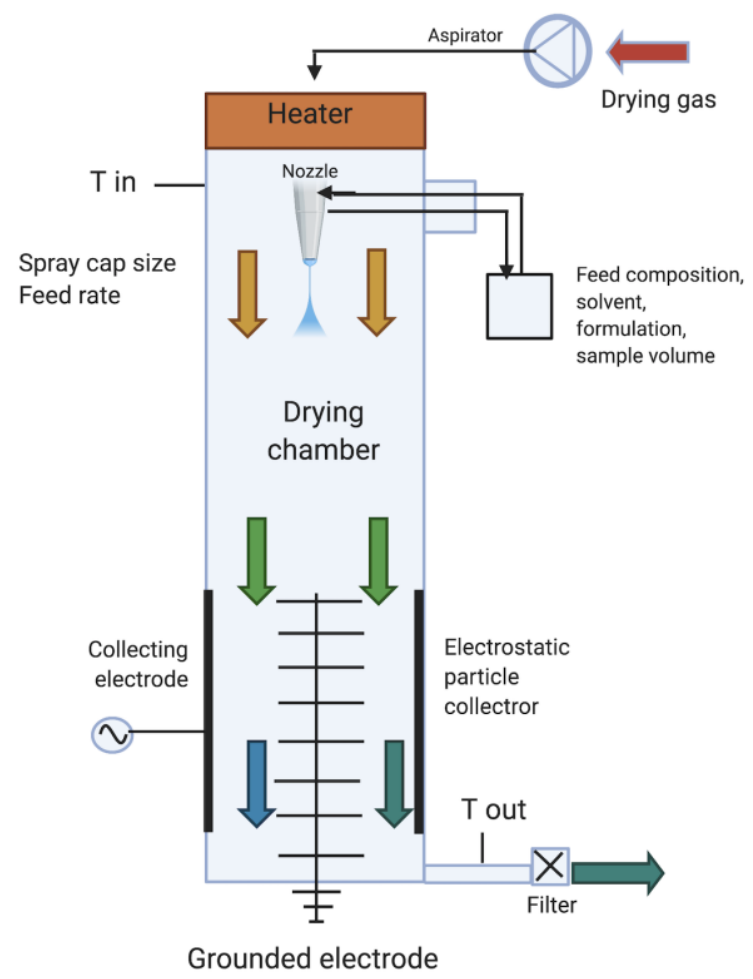

Figure 2. Principle operation of Nanospray drier (Adapted from Arpagaus, 2012) 
The advantages of nanospray drying are 1) particle size and shape optimization for pulmonary drug delivery, 2) high bioavailability of the drug in the lungs as compared to oral administration, 3) reduction of drug doses, 4) minimizing systemic side effects, and 5) increases patient adherence (Arpagaus, 2012, 2019b; Arpagaus et al., 2018). Besides that, nano spray drying is suited for heat-sensitive drugs and various excipients (Haggag \& Faheem, 2015). It also has been used to encapsulate both hydrophobic or hydrophilic drugs, peptides, and proteins into particles for controlled drug release (Swider et al., 2018). A reasonable size range (approximately 0.5-3.3 um) can also be achieved especially for locally acting inhaled drug particles (Arpagaus \& Meuri, 2010). An amount of powder about $30 \mathrm{mg}$ can be collected from the electrostatic particles collector thus allowing economical uses of expensive pharmaceuticals ingredients. Small sample quantities of about 5 to $10 \mathrm{~mL}$ are also possible to be spray dried with the help of a nanospray drying technique (Arpagaus, 2012, 2019b).

\section{Proposed Strategies}

There is a high expectation of using remdesivir and hydroxychloroquine as an effective treatment against SARS-CoV-2 infection due to its promising anti-SARSCoV-2 activity; antiviral, anti-inflammatory, and immunomodulator. A targeted administration of nasal HCQ has been reported to reduce histamine and leukotriene release in one of the animal studies with no significant acute toxicity (Barrett et al., 2008), and in cell cultures and animal model, remdesivir has shown the inhibition of replication of coronavirus. Besides, its proven safety profile and low price in the market make it a drug of choice to be produced on a large-scale amid the SARS-CoV-2 pandemic outbreak. Nanospray inhalation of remdesivir and hydroxychloroquine might be the best option to deliver the drug quickly to the lung epithelial cells particularly, the alveolar regions, where ACE- 2 receptors are mostly located.

The ability of a drug to deposit and retain on the lung epithelial cells, and to overcome extracellular and cellular pulmonary barriers are essential factors to be considered in the design of inhaled powder particles (Ungaro et al., 2012). The capability of the powder particles to deposit on the lung relies on its aerodynamic diameter (Daer) while rapid macrophage uptake and clearance of noxious particles (including solid drug particles) depend on the Dgeo. To get to the deep lung, powder particles should have a Daer and Dgeo below $5 \mu \mathrm{m}$. However, the $5 \mu \mathrm{m}$ of geometric diameter (Dgeo) of the particles can promote aggressive pulmonary macrophage activity, thus resulting in a brief period of delivery for an inhaled drug (Karathanasis et al., 2005). In contrast, nanoparticles with a size $<1 \mu \mathrm{m}$ will have difficulty to deposit at the pulmonary airways as they are mostly will be exhaled after inhalation (Beck-Broichsitter et al., 2012).

To overcome this disadvantage, it is highly recommended to utilise polymeric drugs that are easy to control and target their delivery to a specific region especially the lungs. A polymer such as biodegradable poly(lactic-co-glycolic-acid) (PLGA) is the most frequently used as a carrier-mediated lung targeting for the delivery of inhalation particles (Ungaro et al., 2012). PLGA co-polymer consists of polylactic acid (PLA) and polyglycolic acid (PGA). It can be easily hydrolysed by body fluids into non-toxic metabolites monomers, lactic acid, and glycolic acid causing minimal systemic toxicity (Arpagaus, 2019b; Bartolucci, 2017; Danhier et al., 2012; Essa et al., 2020; Pandey \& Jain, 2015; Swider et al., 2018). It is available for commercial use and licensed by the United States Food and Drug Administration (US FDA) and the European Medicine Agency (EMA) at different molecular weights and lactide/glycolide ratios (Bartolucci, 2017; Danhier et al., 2012; Hirenkumar \& Steven, 2012; Pandey \& Jain, 2015). Interestingly, PLGA copolymer can be stored in powder form for a lengthy period (Swider et al., 2018). Several studies found that nanospray dried PLGA particles could produce from approximately $2 \mu \mathrm{m}$ to below $200 \mathrm{~nm}$ spherical powder particles (Arpagaus, 2019a) which is within an ideal nanoparticle size for use in dry powder inhaler (DPI) (Bartolucci, 2017; Beck-Broichsitter et al., 2012).

By applying the nanospray drying technique, any hydrophobic or hydrophilic active ingredients can be encapsulated in polymeric wall materials to improve the drug formulation and to protect and deliver the drug to the correct site and time in the body (Arpagaus, 2018). Generally, encapsulated nanoparticles are described as solid particles (size less than $1 \mu \mathrm{m}$ ) suspended in a liquid medium. The smaller the size of the nanoparticles, the larger their surface area. Subsequently, the dissolution and absorption rate of the nanoparticles is improved, and thus, lead to the high bioavailability of the encapsulated drug (Arpagaus, 2019b). The common method for PLGAbased nanoparticles formulation that is suitable for hydrophobic drugs is the emulsification-solvent evaporation technique (Danhier et al., 2012; Hirenkumar $\&$ Steven, 2012). In this technique, an organic solvent like dichloromethane will be used to dissolve both polymer and the desired drug. After that, the polymer solution will be mixed with water and a surfactant such as polysorbate- 80 or poloxamer-188 to form an oil in water $(\mathrm{O} / \mathrm{W})$ emulsion (Danhier et al., 2012). The mixture then is atomised into hot gas to evaporate the solvent. The resulting dried particles that contain the drug will be dispersed in an amorphous polymer matrix with a geometric mean diameter of the particles of about 1-2.7 $\mu \mathrm{m}$ and a mean mass diameter of $<4 \mu \mathrm{m}$, (using $4 \mu \mathrm{m}$ spray mesh size) (Arpagaus, 2018). The size range is ideal for the delivery of the drug to the lower respiratory region. 
Several formulation parameters need to be considered to prepare an ideal PLGA-based nanoparticle. The PLGA molecular weight and the ratio of poly-lactic acid to polyglycolic acid can be manipulated to obtain the desired degradation rate and mechanical strength (Arpagaus, 2019b; Danhier et al., 2012; Pandey \& Jain, 2015). The higher molecular weight of PLGA possesses more structural stability, therefore it degrades slowly in in-vivo (Arpagaus, 2019b; Essa et al., 2020). To modify the degradation rate of the polymer, different end-capped functional groups also can be used. It has been shown that ester end-capped polymers degrade slower than acid-end capped polymers, thus, useful for slower release applications (Essa et al., 2020). PLA is rigid, and hydrophobic because of methyl groups' existence. Meanwhile, PGA is more flexible and less hydrophobic (Blasi, 2019; Essa et al., 2020). Therefore, PLGA copolymers that contain a high proportion of lactic acid are hydrophobic, making them absorb water slightly, and degrade more gradually than PLGA that contains a high amount of glycolic acid (Blasi, 2019; Pandey \& Jain, 2015).

PLGA copolymers that made up of D,L-PLA, and D,LPGA are amorphous and allow the embedded active ingredients to disperse more homogenously than in the semi-crystalline form of copolymers which comprised of L-PLA and L-PGA) (Blasi, 2019; Hirenkumar \& Steven, 2012; Pandey \& Jain, 2015). For this amorphous state of PLGA, the important physical-chemical property is the glass transition temperature $(T g)$. PLGA polymer at a rubbery state is vulnerable to chemical and physical changes which in turn could influence the release mechanisms and kinetics of the embedded active ingredients. The $T g$ decreases in conditions such as 1) low lactic acid content, 2) low PLGA molecular weight, and 3) addition of compounds such as excipients or active ingredients (Blasi, 2019). An amorphous form of PLGA co-polymers that consists of $70 \%$ lactic acid content is believed to be suitable for drug delivery application (Essa et al., 2020).

The surface of PLGA nanocarriers can be modulated to prevent it from being recognised and phagocytosed by the pulmonary macrophages. Polyethylene glycol (PEG) and chitosan (natural polymer) are commonly used copolymer coated onto the surface of the PLGA carrier to shields it from being taken up by opsonins (an antibody that makes foreign particles susceptible to phagocytosis) and promotes stronger cellular interaction and retention (Danhier et al., 2012). The addition of surfactant in the polymeric solution (polyvinyl alcohol (PVA), polysorbate 80,60 , and 20 , poloxamer or poloxamine) is suggested to prevent particle aggregation and optimise the colloidal stability by increasing miscibility and dispersion of drugs in the polymeric solution (Beck-Broichsitter et al., 2012; Essa et al., 2020). Besides, a combination of PLGA-based nanoparticles with ligands that specifically bind to receptors on the cell of interest, enables the PLGA carrier to enter the cell by receptor-mediated endocytosis (Essa et al., 2020). This strategy could enhance the targeted delivery systems of the PLGA nanoparticles to the targeted site of action.

For the spray parameters, the preferred organic solvents used in the nanospray drying of PLGA polymers are dichloromethane (DCM), acetone, acetonitrile, ethyl acetate, and mixtures of DCM/methanol. The boiling point of DCM and acetone which is $40^{\circ} \mathrm{C}$ and $56^{\circ} \mathrm{C}$ respectively has allowed a low drying temperature which results in a fast-drying process and hampers particles from binding to or agglomerating on the walls. The ideal inlet drying gas temperature for PLGA dissolved in DCM lies between $29^{\circ}$ and $32^{\circ} \mathrm{C}$ (Arpagaus, 2018, 2019b; Schafroth et al., 2012), meanwhile, the outlet temperature is between $21^{\circ} \mathrm{C}$ and $35^{\circ} \mathrm{C}$, which is below the glass transition temperature of most PLGA polymers (about between $37^{\circ} \mathrm{C}$ and $54^{\circ} \mathrm{C}$ ) (Arpagaus, 2019a; Hirenkumar \& Steven, 2012). The outlet temperature must not exceed the PLGA biopolymer glass transition temperature to avoid softening and yield reduction (Arpagaus, 2019b). Therefore, heat-sensitive pharmaceuticals are the most suitable materials to be used in nanospray drying (Amsalem et al., 2017; Arpagaus, 2019a).

The slow and gentle drying produces an almost smooth and spherical surface of compact carrier particles (Ahmad et al., 2015; Arpagaus \& Meuri, 2010). However, the smooth surface of the carrier particles may result in the generation of auto-adhesive layers surrounding the carrier particles. Consequently, the micronised drug particles could agglomerate thus hinders their detachment during inhalation. To improve the aggregation and disaggregation of the micronised powder particles, chitosan may be used as a co-polymer as it gives a rougher surface (groove space) onto the carrier particle which is a good space to bind a drug and help it spread in the oral cavities (better aerosolisation properties) (Ahmad et al., 2015).

A diluted polymer solution with solids concentration ranging from 0.1 to $1 \%(\mathrm{w} / \mathrm{v})$ could produce spray-dried particles down to a size of $100 \mathrm{~nm}$ (Arpagaus, 2018; Arpagaus et al., 2018). Feed rates depend on the spray cap membrane's size, type of formulation, temperature inlet and spray rate. The feed rate must be adjusted to within a range of $10 \mathrm{~mL} / \mathrm{h}$ to $16 \mathrm{~mL} / \mathrm{h}$ when using a $4.0 \mathrm{uM}$ spray cap (Arpagaus, 2012). Typically, Inert gases such as nitrogen and carbon dioxide are used as drying gases to avoid a blast of explosives from occurring. The concentration is adjusted to be less than $4 \%$ and the inert gases are recirculated entirely within the circuit (Arpagaus et al., 2018). The nozzle spray pretreated with a surfactant solution (leucine) could generate a free-flowing powder with a satisfactory aerosol functioning. This could be 
beneficial for the nanospray dryer to produce efficient powder for inhalation (Patrizia et al., 2014). The encapsulated product must be kept under a controlled condition and a stabilizer may be added into the feed formulation to maintain its activity after nanospray drying. Because most nanosprays dried powders are amorphous after a brief drying time, it is important to store the powders under dry conditions to prevent recrystallization (Arpagaus, 2019b; Arpagaus et al., 2018; Arpagaus \& Meuri, 2010).

\section{Conclusion}

Nano spray drying technology is economically simple, easy to use, and very efficient in the formulation of nanospray inhalation. PLGA has several advantages to be used in nanospray inhalation according to its properties such as biodegradable, biosafety, biocompatibility, versatility in formulation and functionalization. The inhaled drugs provide a rapid and direct local effect on the respiratory regions as compared to oral drugs, suggesting its beneficial uses in diseases associated with respiratory disorders. Several formulation parameters (hydrophilicity/hydrophobicity of drug, polymer molecular weight, polymer composition, and degradation rate) and spraying parameters (spray mesh diameter, spray rate, sample concentration, sample flow rate, drying gas temperature, solvent types, selection of excipients, stabilizers, or surfactants) are important to take into considerations as they have a great impact on the particle size, production yield, encapsulation efficiency, solid-state solubility, and controlled release profiles of the engineered nanoparticles. The demand for large scale production of powder particles may increase especially during the pandemic of the severe acute respiratory syndrome (SARS) caused by coronaviruses. Future research should, therefore, focus on a pilot- and industrial-scale commercialization of this technology to promote the use of nanospray inhalation for the treatment of SARS coronaviruses infection.

\section{Acknowledgements}

The authors are thankful to the International Islamic University Malaysia for funding this work via Grant No. P-RIGS18-028-0028.

\section{Conflict of Interest}

The authors declare no conflict of interest.

\section{References}

Ahmad, M. I., Ungphaiboon, S., \& Srichana, T. (2015). The development of dimple-shaped chitosan carrier for ethambutol dihydrochloride dry powder inhaler. Drug Development and Industrial Pharmacy, 41(5), 791800. https://doi.org/10.3109/03639045.2014.903493
Al-Tawfiq, J. A., Al-Homoud, A. H., \& Memish, Z. A. (2020). Remdesivir as a possible therapeutic option for the COVID-19. Travel Medicine and Infectious Disease, 101615. https://doi.org/10.1016/j.tmaid.2020.101615

Amsalem, O., Nassar, T., Benhamron, S., Lazarovici, P., Benita, S., \& Yavin, E. (2017). Solid nano-innanoparticles for potential delivery of siRNA. Journal of Controlled Release, 257, 144-155. https://doi.org/10.1016/j.jconrel.2016.05.043

Arpagaus, C. (2012). A Novel Laboratory-Scale Spray Dryer to Produce Nanoparticles. Drying Technology, 30(10), 1113-1121. https://doi.org/10.1080/07373937.2012.686949

Arpagaus, C. (2018). Pharmaceutical Particle Engineering via Nano Spray Drying - Process Parameters and Application Examples on the Laboratory-Scale. International Journal of Medical Nano Research, 5(1), 1-15. https://doi.org/10.23937/2378-3664.1410026

Arpagaus, C. (2019a). Nanoparticles of PLA / PLGA Biopolymers prepared by Nano Spray Drying. Proceedings of Eurodrying, 10-12(July), 1-9.

Arpagaus, C. (2019b). PLA/PLGA nanoparticles prepared by nano spray drying. Journal of Pharmaceutical Investigation, 49(4), 405-426. https://doi.org/10.1007/s40005-019-00441-3

Arpagaus, C., Collenberg, A., Rütti, D., Assadpour, E., \& Jafari, S. M. (2018). Nano spray drying for encapsulation of pharmaceuticals. International Journal of Pharmaceutics, 546(1-2), 194-214. https://doi.org/10.1016/j.ijpharm.2018.05.037

Arpagaus, C., \& Meuri, M. (2010). Laboratory Scale Spray Drying of Inhalable Drugs: A Review. Respiratory Drug Delivery, 59, 1-8.

Barrett, E. G., Rudolp, K., Raff, H., \& Charous, B. L. (2008). Nasal hydroxychloroquine (HCQ) decreases nasal congestion and inhibits mediator release after regweed challenge in sensitized beagle dogs. Journal of Allergy and Clinical Immunology, 121(2), S277. https://doi.org/10.1016/j.jaci.2010.12.881

Bartolucci, M. J. (2017). A Poly (Lactic Co Glycolic) Acid Based Dry Powder Inhaler for the Treatment of Pulmonary Tuberculosis. (Master Thesis, Harvard University). Harvard Library. Retrieved from http://nrs.harvard.edu/urn3:HUL.InstRepos:37736755 
Beck-Broichsitter, M., Schweiger, C., Schmehl, T., Gessler, T., Seeger, W., \& Kissel, T. (2012). Characterization of novel spray-dried polymeric particles for controlled pulmonary drug delivery. Journal of Controlled Release, 158(2), 329-335. https://doi.org/10.1016/j.jconrel.2011.10.030

Blasi, P. (2019). Poly(lactic acid)/poly(lactic-co-glycolic acid)-based microparticles: an overview. Journal of Pharmaceutical Investigation, 49(4), 337-346. https://doi.org/10.1007/s40005-019-00453-z

Bouadma, L., Lescure, F. X., Lucet, J. C., Yazdanpanah, Y., \& Timsit, J. F. (2020). Severe SARS-CoV-2 infections: practical considerations and management strategy for intensivists. Intensive Care Medicine, 46(4), 579-582. https://doi.org/10.1007/s00134-020-05967-x

Colson, P., Rolain, J. M., \& Raoult, D. (2020). Chloroquine for the 2019 novel coronavirus SARS-CoV-2. International Journal of Antimicrobial Agents. https://doi.org/10.1016/j.ijantimicag.2020.105923

Covid-19 Visualiser. (2020). Retrieved from http://www.covidvisualizer.com

Danhier, F., Ansorena, E., Silva, J. M., Coco, R., Le Breton, A., \& Préat, V. (2012). PLGA-based nanoparticles: An overview of biomedical applications. Journal of Controlled Release, 161(2), 505-522. https://doi.org/10.1016/j.jconrel.2012.01.043

Eastman, R. T., Roth, J. S., Brimacombe, K. R., Simeonov, A., Shen, M., Patnaik, S., \& Hall, M. D. (2020). Remdesivir: A review of its discovery and development leading to human clinical trials for treatment of COVID-19. Preprints, April, 1-20. https://doi.org/10.20944/preprints201810.0478.v1

Essa, D., Kondiah, P. P. D., Choonara, Y. E., \& Pillay, V. (2020). The Design of Poly(lactide-co-glycolide) Nanocarriers for Medical Applications. Frontiers in Bioengineering and Biotechnology, 8(February), 120. https://doi.org/10.3389/fbioe.2020.00048

Gao, J., Tian, Z., \& Yang, X. (2020). Breakthrough: Chloroquine phosphate has shown apparent efficacy in treatment of COVID-19 associated pneumonia in clinical studies. BioScience Trends, 14(1), 72-73. https://doi.org/10.5582/BST.2020.01047

Gaurav, J., \& Ramarao, P. (2020). Virtual Screening Enabled Selection of Antiviral Agents Against Covid19 Disease Targeting Coronavirus Endoribonuclease NendoU: Plausible Mechanistic Interventions in the Treatment of New Virus Strain. ChemRxiv, 00876, 122. https://doi.org/10.26434/chemrxiv.12198966.v1

Gordon, C. J., Tchesnokov, E. P., Woolner, E., Perry, J. K., Feng, J. Y., Porter, D. P., \& Gotte, M. (2020). Remdesivir is a direct-acting antiviral that inhibits
RNA-dependent RNA polymerase from severe acute respiratory syndrome coronavirus 2 with high potency. Journal of Biological Chemistry, 1-26. https://doi.org/10.1074/jbc.ra120.013679

Guo, Y. R., Cao, Q. D., Hong, Z. S., Tan, Y. Y., Chen, S. D., Jin, H. J., ...Yan, Y. (2020). The origin, transmission and clinical therapies on coronavirus disease 2019 (COVID-19) outbreak- An update on the status. Military Medical Research, 7(1), 1-10. https://doi.org/10.1186/s40779-020-00240-0

Haggag, Y. A., \& Faheem, A. M. (2015). Evaluation of nano spray drying as a method for drying and formulation of therapeutic peptides and proteins. Frontiers in Pharmacology, 6(JUL), 1-5. https://doi.org/10.3389/fphar.2015.00140

Hirenkumar, M., \& Steven, S. (2012). Poly Lactic-coGlycolic Acid (PLGA) as Biodegradable Controlled Drug Delivery Carrier. Polymers, 3(3), 1-19. https://doi.org/10.3390/polym3031377.Poly

Holshue, M. L., DeBolt, C., Lindquist, S., Lofy, K. H., Wiesman, J., Bruce, H., ... Pillai, S. K. (2020). First case of 2019 novel coronavirus in the United States. New England Journal of Medicine, 382(10), 929-936. https://doi.org/10.1056/NEJMoa2001191

Itani, R., Tobaiqy, M., \& Faraj, A. Al. (2020). Optimizing use of theranostic nanoparticles as a life-saving strategy for treating COVID-19 patients. Theranostics, 10(13), 5932-5942. https://doi.org/10.7150/thno.46691

Karathanasis, E., Ayyagari, A. L., Bhavane, R., Bellamkonda, R. V., \& Annapragada, A. V. (2005). Preparation of in vivo cleavable agglomerated liposomes suitable for modulated pulmonary drug delivery. Journal of Controlled Release, 103(1), 159175. https://doi.org/10.1016/j.jconrel.2004.11.009

Ko, W., Rolain, J., Lee, N., Chen, P., \& Huang, C. (2020). Arguments in favour of remdesivir for treating SARSCoV-2 infections.

International Journal of Antimicrobial Agents, 55(4), 105933.

https://doi.org/10/1016/j.ijantimicag.2020.105933

Liu, J., Cao, R., Xu, M., Wang, X., Zhang, H., Hu, H., ... Wang, M. (2020). Hydroxychloroquine, a less toxic derivative of chloroquine, is effective in inhibiting SARS-CoV-2 infection in vitro. Cell Discovery, 6(1), 6-9. https://doi.org/10.1038/s41421-020-0156-0

Moore, N. (2020). Chloroquine for COVID-19 Infection. Drug Safety, 43(5), 393-394. https://doi.org/10.1007/s40264-020-00933-4 
Morse, J. S., Lalonde, T., Xu, S., \& Liu, W. R. (2020). Learning from the Past: Possible Urgent Prevention and Treatment Options for Severe Acute Respiratory Infections Caused by 2019-nCoV. ChemBioChem, 21(5), 730-738. https://doi.org/10.1002/cbic.202000047

O’Neill, P. M., Bray, P. G., Hawley, S. R., Ward, S. A., \& Kevin Park, B. (1998). 4-Aminoquinolines - Past, present, and future: A chemical perspective. Pharmacology and Therapeutics, 77(1), 29-58. https://doi.org/10.1016/S0163-7258(97)00084-3

Panati, K., \& Narala, V. R. (2020). COVID-19 Outbreak: an Update on Therapeutic Options. SN Comprehensive Clinical Medicine, 3-4. https://doi.org/10.1007/s42399-020-00264-6

Pandey, A., \& Jain, D. S. (2015). Poly Lactic-Co-Glycolic Acid (PLGA) Copolymer and Its Pharmaceutical Application. Handbook of Polymers for Pharmaceutical Technologies, 2, 151-172. https://doi.org/10.1002/9781119041412.ch6

Patrizia, A. R., Mariateresa, S., Pasquale, D. G., Teresa, M., Francesca, S., \& Paola, R. (2014). Nanospray Drying as a Novel Technique for the Manufacturing of Inhalable NSAID Powders. Scientific World Journal, 2014, 1-8. https://doi.org/10.1155/2014/838410

Schafroth, N., Arpagaus, C., Jadhav, U. Y., Makne, S., \& Douroumis, D. (2012). Nano and microparticle engineering of water insoluble drugs using a novel spray-drying process. Colloids and Surfaces B: Biointerfaces, $90(1), \quad 8-15$. https://doi.org/10.1016/j.colsurfb.2011.09.038

She, J., Jiang, J., Ye, L., Hu, L., Bai, C., \& Song, Y. (2020). 2019 novel coronavirus of pneumonia in Wuhan, China: emerging attack and management strategies. Clinical and Translational Medicine, 9(1). https://doi.org/10.1186/s40169-020-00271-z

Sheahan, T. P., Sims, A. C., Graham, R. L., Menachery, V. D., Gralinski, L. E., Case, J. B.,... Baric, R. S. (2017). Broad-spectrum antiviral GS-5734 inhibits both epidemic and zoonotic coronaviruses. Science Translational Medicine, 9(396), 1-11. https://doi.org/10.1126/scitranslmed.aal3653

Singh, A. K., Singh, A., Shaikh, A., Singh, R., \& Misra, A. (2020). Chloroquine and hydroxychloroquine in the treatment of COVID-19 with or without diabetes: A systematic search and a narrative review with a special reference to India and other developing countries. Diabetes and Metabolic Syndrome: Clinical Research and Reviews, 14(3), 241-246. https://doi.org/10.1016/j.dsx.2020.03.011
Swider, E., Koshkina, O., Tel, J., Cruz, L. J., de Vries, I. J. M., \& Srinivas, M. (2018). Customizing poly(lacticco-glycolic acid) particles for biomedical applications. Acta Biomaterialia, 73, 38-51. https://doi.org/10.1016/j.actbio.2018.04.006

Thomé, R., Lopes, S. C. P., Costa, F. T. M., \& Verinaud, L. (2013). Chloroquine: Modes of action of an undervalued drug. Immunology Letters, 153(1-2), 5057. https://doi.org/10.1016/j.imlet.2013.07.004

Ungaro, F., D’Angelo, I., Miro, A., La Rotonda, M. I., \& Quaglia, F. (2012). Engineered PLGA nano- and micro-carriers for pulmonary delivery: Challenges and promises. Journal of Pharmacy and Pharmacology, 64(9), 1217-1235. https://doi.org/10.1111/j.20427158.2012.01486.x

Wang, M., Cao, R., Zhang, L., Yang, X., Liu, J., Xu, M., ... Xiao, G. (2020). Remdesivir and chloroquine effectively inhibit the recently emerged novel coronavirus (2019-nCoV) in vitro. Cell Research, 30(3), 269-271. https://doi.org/10.1038/s41422-020$0282-0$

Warhurst, D. C., Steele, J. C. P., Adagu, I. S., Craig, J. C., \& Cullander, C. (2003). Hydroxychloroquine is much less active than chloroquine against chloroquineresistant Plasmodium falciparum, in agreement with its physicochemical properties. Journal of Antimicrobial Chemotherapy, 52(2), 188-193. https://doi.org/10.1093/jac/dkg319

Yang, P., \& Wang, X. (2020). COVID-19: a new challenge for human beings. Cellular and Molecular Immunology, March, 19-21. https://doi.org/10.1038/s41423-020-0407-x

Zhang, T., He, Y., Xu, W., Ma, A., Yang, Y., \& Xu, K. F. (2020). Clinical trials for the treatment of Coronavirus disease 2019 (COVID-19): A rapid response to urgent need. Science China Life Sciences, 63(5), 774-776. https://doi.org/10.1007/s11427-020-1660-2

Zhou, G., Chen, S., \& Chen, Z. (2020a). Back to the spring of Wuhan: facts and hope of COVID-19 outbreak. Frontiers of Medicine, 1-4. https://doi.org/10.1007/s11684-020-0758-9

Zhou, M., Zhang, X., \& Qu, J. (2020). Coronavirus disease 2019 (COVID-19): a clinical update. Frontiers of Medicine, 2019. https://doi.org/10.1007/s11684-0200767-8 